\title{
Smallest limited snakes
}

\author{
László Szabó and Zoltán Ujváry-Menyhárt
}

László Szabó was born in Budapest in 1966. He studied mathematics and computer science at Eötvös Loránd University and received his Ph.D. in mathematics from the Hungarian Academy of Sciences in 1996. His research interests are discrete geometry and convexity.

Zoltán Ujváry-Menyhárt was born in Budapest in 1973. He studied mathematics at Eötvös Loránd University and computer science at Budapest University of Technology and Economics. His research interests are combinatorics and discrete geometry.

\section{Introduction}

A (topological) disk is a subset of the euclidean plane homeomorphic to the unit ball. If two disks have a common interior point then we say that the disks overlap. A sequence $\mathcal{C}=\left\langle C_{1}, \ldots, C_{n}\right\rangle$ of mutually non overlapping congruent disks where $C_{i} \cap C_{j} \neq \emptyset$ if and only if $|i-j| \leq 1$ is called a snake. If the snake $\mathcal{C}$ is not a proper subset of another snake of disks congruent to the members of $\mathcal{C}$ then we say that the snake is limited.

We are concerned with the following question: What is the minimum number of mutually non overlapping congruent disks which can form a limited snake? Here we prove

Theorem. The minimum number of mutually non overlapping congruent disks which can form a limited snake is four.

Surprisingly, under the assumption of convexity the above problem seems to be much more complicated. Fig. 1 shows that six mutually non overlapping congruent copies of a

Auf einem Tisch legt man mit lauter gleichen Münzen eine „Münzschlange“: an eine erste Münze anstossend legt man eine zweite, daran anstossend eine dritte usw. Bei diesem Legespiel kann eine Konfiguration entstehen, bei der man weder am Kopf noch am Schwanz der Schlange eine weitere Münze anschliessen kann, weil der Platz durch andere Münzen des Schlangenkörpers versperrt wird. Welches ist die kleinste Anzahl Münzen, bei der dies vorkommen kann? Die Autoren untersuchen und beantworten die entsprechende Frage, wenn man die runden Münzen durch eine beliebige einfach zusammenhängende beschränkte Menge der Euklidischen Ebene ersetzt. Das entsprechende Problem für konvexe beschränkte Mengen ist hingegen noch offen. 
certain rectangle can form a limited snake. Do there exist convex disks whose less then six mutually non overlapping congruent copies could form limited snakes? We conjecture that the answer to this question is in the negative.

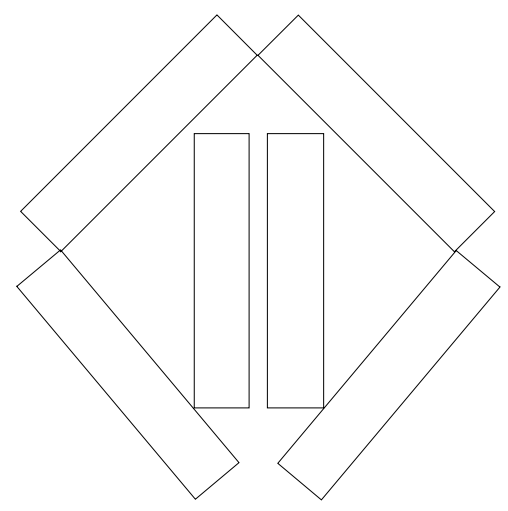

Fig. 1

Also, the problem of determining the minimum number of mutually non overlapping congruent copies of a given disk which can form a limited snake is very complicated. The only known result in this direction is that the minimum number of mutually non overlapping congruent balls which can form a limited snake is ten (see [1]).

For additional results on more restrictive variants of the snake problem, see $[2,3,4,5,6$, $7,8]$.

\section{Proof of the theorem}

Fig. 2 shows that this minimum number is at most four.

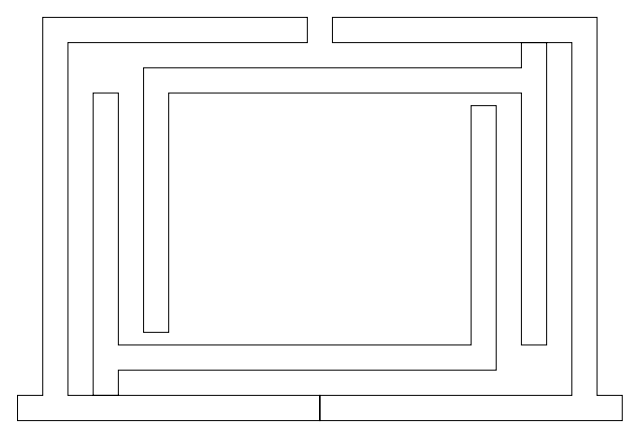

Fig. 2

To complete the proof we have to show that two or three mutually non overlapping congruent disks cannot form a limited snake. Let $C$ be an arbitrary disk and let $\mathcal{C}=\left\langle C_{1}, \ldots, C_{n}\right\rangle$ be a limited snake consisting of disks congruent with $C$. 
We start with the case $n=2$. First assume that $\operatorname{conv} C_{1}=\operatorname{conv} C_{2}$, i.e., the convex hulls of $C_{1}$ and $C_{2}$ coincide. If every boundary point of $\operatorname{conv} C_{1}$ belongs to $C_{1}$, i.e., $C_{1}$ is convex, then $C_{1}$ and $C_{2}$ coincide, which is impossible. Thus there exists a boundary point of conv $C_{1}$ which does not belong to $C_{1}$. This point lies in the relative interior of a segment joining two extreme points, say $A$ and $B$, of $\operatorname{conv} C_{1}$. Recall that a point of a disk is an extreme point of the disk if there exists no segment in the disk that contains the point in its relative interior. The points $A$ and $B$ are extreme points of $C_{1}$ and they can be joined with a path $P_{1}$ whose points different from $A$ and $B$ lie in the interior of $C_{1}$. Also, the points $A$ and $B$ are extreme points of $C_{2}$ and they can be joined with a path $P_{2}$ whose points different from $A$ and $B$ lie in the interior of $C_{2}$. Then either the bounded region surrounded by $P_{1}$ and the segment $\overline{A B}$ contains $P_{2}$ or the bounded region surrounded by $P_{2}$ and the segment $\overline{A B}$ contains $P_{1}$, which is impossible since $\operatorname{conv} C_{1}=\operatorname{conv} C_{2}$.

Thus there exists a point $P$ of $C_{1}$ which does not belong to conv $C_{2}$. Then $P$ can be strictly separated from $C_{2}$ by a line $l$. Let $l^{\prime}$ be the support line of $C_{1}$ which is parallel to $l$ and does not separate $C_{1}$ and $C_{2}$. Reflecting $C_{1}$ with respect to $l^{\prime}$ we obtain a third copy of $C$ which forms with $C_{1}$ and $C_{2}$ a three element snake, a contradiction.

Now we turn to the case $n=3$. Let $\overline{D E}$ be a diameter of $C_{1}$ and consider the stripe $S_{1}$ whose boundary lines, say $l_{1}$ and $l_{2}$, go through $D$ and $E$, respectively, and are perpendicular to $\overline{D E}$. If $C_{3}$ is not contained in $S_{1}$ then consider the support line $l$ of $C_{3}$ which is parallel to $l_{1}$ and whose distance from $S_{1}$ is maximal. Without loss of generality we may assume that $l_{2}$ separates $l$ and $l_{1}$. Let $F$ be a common point of $C_{3}$ and $l$. The disk $C_{2}$ cannot intersect both $l$ and $l_{1}$ since the distance between the two lines is greater than the diameter of $C_{2}$. Thus either reflecting $C_{1}$ with respect to $l_{1}$ or reflecting $C_{3}$ with respect to $l$ we obtain a fourth copy of $C$ which forms with $C_{1}, C_{2}$ and $C_{3}$ a four element snake, a contradiction.

Thus $C_{3}$ lies in $S_{1}$. Let $\overline{G H}$ be a diameter of $C_{3}$ and consider the stripe $S_{3}$ whose boundary lines, say $l_{3}$ and $l_{4}$, go through $G$ and $H$, respectively, and are perpendicular to $\overline{G H}$. If $C_{1}$ is not contained in $S_{3}$ then repeating the previous argument we obtain a contradiction. Therefore $C_{1}$ lies in $S_{3}$. If $S_{1}=S_{3}$, i.e., $l_{1}=l_{3}$ and $l_{2}=l_{4}$ without loss of generality, then $D$ and $G$ are different points since $C_{1} \cap C_{3}=\emptyset$. Now $C_{2}$ does not contain both $D$ and $H$ since their distance is greater than the diameter of $C_{2}$. Therefore either reflecting $C_{1}$ with respect to $l_{1}$ or reflecting $C_{3}$ with respect to $l_{2}$ we obtain a fourth copy of $C$ which forms with $C_{1}, C_{2}$ and $C_{3}$ a four element snake, a contradiction. On the other hand, if $S_{1}$ and $S_{2}$ are different stripes then their intersection is a parallelogram which contains both $C_{1}$ and $C_{3}$. The points $D$ and $E$ can be joined by a path $P_{3}$ in $C_{1}$ while $G$ and $H$ can be joined by a path $P_{4}$ in $C_{3}$. Since the pathes join opposite sides of the above parallelogram they necessarily intersect each other. But this is impossible since $C_{1}$ and $C_{3}$ are disjoint. This completes the proof of the theorem.

\section{References}

[1] Bisztriczky, T.; Böröczky Jr, K.; Harborth, H.; Piepmeyer, L.: On the smallest limited snake of unit disks. Geom. Dedicata 40 (1991), 319-324.

[2] Bisztriczky, T.; Harborth, H.: Smallest limited edge-to-edge snakes in Euclidean tessellations. Congr. Numer. 149 (2001), 155-159. 
[3] Böröczky Jr, K.; Soltan, V.: Smallest maximal snakes of translates of convex domains. Geom. Dedicata 54 (1995), 31-44.

[4] Harborth, H.: Problem 45: Kleinste endliche Schlange. Math. Semesterber. 36 (1989), 269-270.

[5] Harborth, H.; Szabó, L.; Ujváry-Menyhárt, Z.: Smallest limited vertex-to-vertex snakes of unit triangles. Geom. Dedicata 78 (1999), 171-181.

[6] Heidelberg, R.; Stege, L.; Weiß, H.: Lösung zu Problem 45. Math. Semesterber. 38 (1991), 137-138.

[7] Hering, F.: Beweis einer Vermutung von Heiko Harborth über Polyominos aus Quadraten. Math. Semesterber. 38 (1991), 223-237.

[8] Szabó, L.; Ujváry-Menyhárt, Z.: Maximal facet-to-facet snakes of unit cubes. Beiträge Algebra Geom. 42 (2001), 203-217.

László Szabó and Zoltán Ujváry-Menyhárt

Computer and Automation Institute

Hungarian Academy of Sciences

Lágymányosi utca 11

H-1111 Budapest, Hungary

e-mail: lszabo@csillag.ilab.sztaki.hu 